\title{
Asymptotic von Neumann measurement strategy for solid-state qubits
}

\author{
F. K. Wilhelm \\ Quantum Transport Group, Technische Natuurkunde, TU Delft, P.O. Box 5046, 2600 GA Delft, The Netherlands \\ and Sektion Physik and CeNS, Ludwig-Maximilians-Universität, Theresienstrasse 37, 80333 München, Germany
}

(Received 19 May 2003; published 19 August 2003)

\begin{abstract}
A measurement on a macroscopic quantum system does not, in general, lead to a projection of the wave function in the basis of the detector as predicted by von Neumann's postulate. Hence, it is a question of fundamental interest, how the preferred basis onto which the state is projected is selected out of the macroscopic Hilbert space of the system. Detector-dominated von Neumann measurements are also desirable for both quantum computation and verification of quantum mechanics on a macroscopic scale. The connection of these questions to the predictions of the spin-boson model is outlined. I propose a measurement strategy, which uses the entanglement of the qubit with a weakly damped harmonic oscillator. It is shown that the degree of entanglement controls the degree of renormalization of the qubit and identify that this is equivalent to the degree to which the measurement is detector dominated. This measurement very rapidly decoheres the initial state, but the thermalization is slow. The implementation in Josephson quantum bits is described and it is shown that this strategy also has practical advantages for the experimental realization.
\end{abstract}

DOI: 10.1103/PhysRevB.68.060503

The field of quantum computation ${ }^{1}$ has been experimentally pioneered in quantum optics, atomic physics, and nuclear magnetic resonance (NMR). In these quantummechanical systems with few degrees of freedom and strong quantum coherence, the measurement devices ("meters") are well described and can be classified into two types. In atomic physics, e.g., "strong" measurements can be performed, which satisfy von Neumann's measurement postulate, ${ }^{2}$ i.e., the state of the system is projected onto the eigenstate of the meter corresponding to the measurement result. In NMR, on the other hand, the meter couples weakly to each individual spin and decoheres it only weakly. In order to still obtain enough signal and information, the measurement is performed on an ensemble of qubits.

These qubits cannot be easily integrated to large-scale circuits. Thus, solid-state qubits, which can be lithographically manufactured, are a promising alternative. Solid-state systems consist of many degrees of freedom, hence quantum coherence can so far only be maintained over very short times. ${ }^{3,4}$ It was proposed that superconducting Josephson circuits in the charge $e^{3,5}$ or flux ${ }^{4,6}$ regime could act as solid-state qubits with appreciable coherence times. In these cases, the measurement apparatus is permanently close to the qubit, although the interaction may effectively be switched off. ${ }^{7,8}$ The measurement process in this system can be described within the spin-boson ${ }^{9,10}$ or related models. ${ }^{7,11,12}$

From a density-matrix description, we can obtain detailed (although incomplete) information about the dynamics of the measurement: After a dephasing time $\tau_{\phi}$, the density matrix is brought into an incoherent mixture, and after the relaxation time $\tau_{\mathrm{r}}$ it thermalizes and the information about the initial state is lost. ${ }^{7}$ In order to render $\tau_{\mathrm{r}}$ long enough, usually $^{3-5}$ the meter is only weakly coupled to the qubit. This makes it necessary to ensemble average by repeating the measurement. Theoretical research ${ }^{7,11,12}$ shows that an optimization of these weak measurements allows for single-shot measurements without averaging, by waiting longer than the dephasing time. These are optimized weak measurements or qubit dominated measurements: They completely decohere
PACS number(s): 03.67.Lx, 03.65.Ta, 03.65.Yz, 74.50.+r

the state of the qubit, however, the final state is not an eigenstate of the measured observable, but of the qubit. Qubit and apparatus do not get strongly entangled. It has also been shown theoretically, ${ }^{7}$ that detector-dominated strong measurements of superconducting qubits are possible, on the expense of $\tau_{\mathrm{R}}$ being very short, which sets a strong experimental challenge. It is a fundamental question, under which conditions a measurement performed on a potentially macroscopic object follows the postulates of quantum mechanics and how, in general, the preferred observable basis is selected out of the large Hilbert space of the system and the detector. ${ }^{13}$ This question should be addressed using specific models which describe actual detectors. Moreover, there are practical issues: (i) The theoretical signal-to-noise ratio of a weak measurement is limited to 4 (Ref. 12), (ii) and Efficient quantum algorithms such as error correction ${ }^{14}$ or the test of Bell-type inequalities ${ }^{15}$ rely on strong measurements.

In this paper, I am going to connect the abstract notions of quantum measurement theory to the concepts of the spinboson model, in particular, the issue of entanglement will be connected to scaling of the tunnel matrix element. I will outline a method how to perform genuine detectordominated measurements in this context.

For definiteness, it is assumed that the variable of the quantum bit which is measured is described by the pseudospin operator $\hat{\sigma}_{\mathrm{z}}$. When the measurement apparatus is coupled to the qubit, the same term experiences a fluctuating force, which is assumed to be Gaussian and be modeled by a bath of harmonic oscillators. Consequently, we end up with the spin-boson Hamiltonian. ${ }^{9,16}$ After integrating out high frequencies, its pseudospin part reads

$$
\hat{H}_{\text {eff }}=\hbar\left(\frac{\epsilon}{2} \hat{\sigma}_{z}+\frac{\Delta_{\text {eff }}}{2} \hat{\sigma}_{x}\right),
$$

where the off-diagonal term $\Delta_{\text {eff }}$ is in general rescaled due to the environment as compared to the original splitting $\Delta$ of an isolated qubit. The spin-boson model generally predicts ${ }^{16}$ the dynamics described in the preceding section. In particular, 
after the dephasing time, the density matrix is projected onto a mixture of eigenstates of $H_{\text {eff. }}$. Usually, in the weakcoupling regime, ${ }^{9} \Delta_{\text {eff }}$ is close to the bare $\Delta$ of the qubit and consequently the eigenstates of $H_{\text {eff }}$ are far from being eigenstates of $\hat{\sigma}_{\mathrm{z}}$. Consequently, the state of the qubit will not be projected onto the measured variable, i.e., the measurement is qubit dominated. A detector-dominated measurement would be realized for $\Delta_{\text {eff }}=0,{ }^{17}$ i.e., when $\hat{H}_{\text {eff }}=(\epsilon / 2) \hat{\sigma}_{\mathrm{z}}$ and hence commutes with the coupling to the meter. A number of schemes allow to directly suppress $\Delta$ using an external control parameter. ${ }^{5,6,18}$ In what follows, I want to describe, using a generic model, how this is accomplished by the measurement apparatus itself, in agreement with the usual understanding of quantum measurements.

Consider a qubit coupled to a single (big) harmonic oscillator, which experiences linear friction, which is in turn described quantum mechanically through a bath of oscillators. The Hamiltonian of this system reads

$$
\begin{aligned}
\hat{H}= & \hbar\left(\frac{\epsilon}{2} \hat{\sigma}_{z}+\frac{\Delta}{2} \hat{\sigma}_{x}\right)+\frac{\hat{P}^{2}}{2 M}+\frac{M}{2} \Omega^{2}\left(\hat{X}-q \hat{\sigma}_{z}\right)^{2} \\
& +\sum_{i}\left(\frac{\hat{p}_{i}^{2}}{2 m_{i}}+\frac{m_{i}}{2} \omega_{i}^{2}\left(\hat{x}_{i}-\left(c_{i} / m_{i} \omega_{i}^{2}\right) \hat{X}\right)^{2}\right),
\end{aligned}
$$

where the displacement $q$ characterizes the coupling of the qubit to the big oscillator. The oscillator bath is characterized through an ohmic spectral density $J(\omega)=\Sigma\left(\pi c_{i}^{2}\right) /$ $\left(2 m_{i} \omega_{i}\right) \delta\left(\omega-\omega_{i}\right)=M \Gamma \omega$, where $\Gamma / 2$ is the friction coefficient of the damped big oscillator. It was shown ${ }^{19}$ that this system is equivalent to the spin-boson model, with an effective spectral density

$$
J_{\mathrm{eff}}(\omega)=2 \pi \alpha \omega \frac{\Omega^{4}}{\left(\omega^{2}-\Omega^{2}\right)^{2}+4 \Gamma^{2} \omega^{2}},
$$

where $\alpha=2 M q^{2} \Gamma / h$ is a dimensionless dissipation coefficient, which here is assumed to be small, $\alpha \ll 1$. From now on, we want to concentrate on the case $\epsilon, \Delta \ll \Omega$.

At $\Gamma=0$, the low-energy Hilbert space is spanned by $| \pm\rangle_{\text {eff }}=| \pm\rangle|L / R\rangle$ where $| \pm\rangle$ are the basis states of the qubit, $\sigma_{z}| \pm\rangle= \pm| \pm\rangle$, and $|L / R\rangle$ are coherent states of the harmonic oscillator centered around $X= \pm q$, see Fig. 1 (left). So in a general low-energy state $|\psi\rangle=a|+\rangle_{\text {eff }}+b|-\rangle_{\text {eff }}$, $|a|^{2}+|b|^{2}=1$, qubit and oscillator are entangled ${ }^{20}$ and the oscillator states are pointers onto the qubit states. ${ }^{13}$ In this low-energy basis, the Hamiltonian acquires form (1), with $\Delta_{\text {eff }}=\Delta\langle L \mid R\rangle=\Delta e^{-\eta}$ where $\eta=M \Omega q^{2} / \hbar$. Under an appropriate choice of parameters, we can achieve $\eta>1$ and $\Delta_{\text {eff }}$ $\ll \Delta$. This choice corresponds to the condition of almost [meaning here and henceforth "up to an error of $O\left(e^{-\eta}\right)$ "] orthogonal states in the environment, which has been identified as the condition for an ideal von Neumann measurement. ${ }^{13,21}$

For finite $\Gamma$, this system can be analyzed using adiabatic renormalization. ${ }^{9,22}$ One finds ${ }^{23} \Delta_{\text {eff,damp }}(\alpha)$ $=\Delta e^{-\eta /(1-\alpha)}(\Delta / \Omega)^{\alpha /(1-\alpha)}$. Thus, finite dissipation $0<\alpha$ $\ll 1$ scales $\Delta$ down even slightly further.

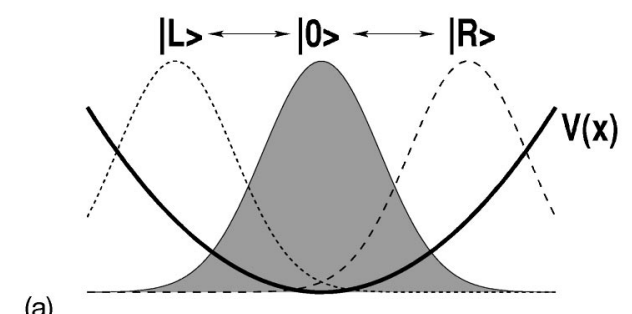

(a)

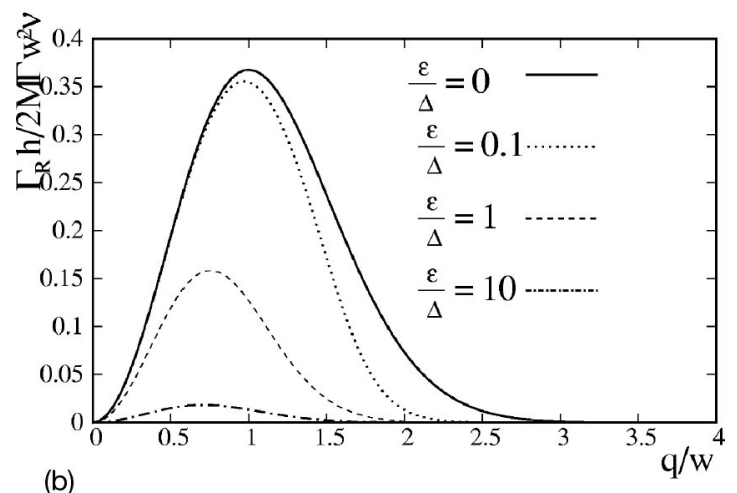

FIG. 1. Left: Visualization of the ground state $|0\rangle$ and the coherent pointer states $|L\rangle$ and $|R\rangle$ of the oscillator in the potential $V(x)$; right: Relaxation rates as a function of the coupling $q / w$ for different energy biases. $w$ is the width of the ground-state wave function of the pointer, $w=\sqrt{\hbar / M \Omega}$.

The coherence properties of our system can at $\epsilon, \Delta, T$ $\ll \Omega$ be studied using a systematic weak damping approximation ${ }^{16}$ of the spin-boson model. The relaxation and dephasing rates $\Gamma_{\mathrm{r} / \phi}=\tau_{\mathrm{r} / \phi}^{-1}$ are given by

$$
\Gamma_{\mathrm{r}}=\pi \alpha \frac{\Delta_{\text {eff }}^{2}}{\nu_{\text {eff }}} \operatorname{coth}\left(\frac{\nu}{2 T}\right), \quad \Gamma_{\phi}=\frac{\Gamma_{\mathrm{r}}}{2}+2 \pi \alpha k_{\mathrm{B}} \frac{\epsilon^{2}}{\nu_{\text {eff }}} T / \hbar,
$$

where $\nu_{\text {eff }}=\sqrt{\Delta_{\text {eff }}^{2}+\epsilon^{2}}$. In our case, if $\eta>1, \Delta_{\text {eff }}$ is exponentially reduced compared to $\Delta$, transitions between the basis states are suppressed leaving relaxation very slow, i.e., the state becomes almost localized or "frozen," see Fig. 1 (right). The second contribution to $\Gamma_{\phi}$ in Eq. (4) reflects dephasing processes which do not change the qubit energy and are consequently not frozen.

The use of a weak damping approximation for $\Gamma_{\mathrm{r}, \phi}$ is appropriate, although $J(\omega)$ can be large at the peak and in fact the down scaling of $\Delta_{\text {eff }}$ is essentially a nonperturbative effect. However, decoherence is mostly probing the $J_{\text {eff }}(\omega)$ around $\omega=\nu_{\text {eff }} \ll \Omega$, where the weak damping condition holds. This is supported by two observations: i) if we project the full Hamiltonian onto its low-energy Hilbert space spanned by $| \pm\rangle_{\text {eff }}$, we find an effective ohmic model leading to Eq. 4; (ii) a full nonperturbative calculation ${ }^{23}$ based on the noninteracting blip approximation (NIBA) ${ }^{9}$ reproduces both the scaling and $\Gamma_{\mathrm{r}}$ within the known ${ }^{16}$ limitations of NIBA.

The measurement can now be performed as follows: As a first step, $q$ is adiabatically ramped from $q=0$ to a finite $q_{0}$ where $\eta>1$ and $\Delta \rightarrow \Delta_{\text {eff }} \ll \Delta$. The adiabatic theorem pre- 
dicts, that the state of the system evolves as $(\alpha|+\rangle$ $+\beta|-\rangle) \otimes|0\rangle \rightarrow\left(\alpha^{\mathrm{eff}}|+\rangle|L\rangle+\beta^{\mathrm{eff}}|-\rangle|R\rangle\right)$, where

$$
\left(\begin{array}{c}
\alpha^{\mathrm{eff}} \\
\beta^{\mathrm{eff}}
\end{array}\right)=\left(\begin{array}{cc}
\cos \left(\frac{\theta^{\mathrm{eff}}-\theta}{2}\right) & \sin \left(\frac{\theta^{\mathrm{eff}}-\theta}{2}\right) \\
-\sin \left(\frac{\theta^{\mathrm{eff}}-\theta}{2}\right) & \cos \left(\frac{\theta^{\text {eff }}-\theta}{2}\right)
\end{array}\right)\left(\begin{array}{c}
\alpha \\
\beta
\end{array}\right)
$$

and $\tan \theta^{\text {(eff) }}=\epsilon / \Delta^{\text {(eff) }}$. The condition for adiabaticity is $d q / d t \ll \nu_{\text {eff }}^{2} / 2 q M \Omega \Delta_{\text {eff }}$, i.e., for small $q$ the ramping can be very fast.

When $\eta>1$, the matrix element is scaled down and the state is "premeasured" by entanglement with well-separated pointer states $L$ and $R \cdot{ }^{13}$ Only now, we start the measurement, by coupling the oscillator to the meter and decohering the state is projected onto the eigenstates of $\hat{H}_{\text {eff }}$, which are close to the ones of $\hat{\sigma}_{z}$. We can then read off the position of the big oscillator serving as a pointer and switch off the meter (or $q$ ) again way before $\tau_{\mathrm{r}}$ without destroying information by relaxation.

In practice, it will usually not be possible to switch the coupling between oscillator and meter separately. Thus, before the entanglement is established, the relaxation rate (4) does not profit from the reduction of $\Delta_{\text {eff }}$, see Fig. 1 (right). In order not to lose the information to be measured, the maximum relaxation rate $\Gamma_{\mathrm{r}, \max }$ reached at $\eta=1 / 2$ (i.e., $q$ $=q_{\mathrm{c}}=\sqrt{\hbar / 2 M \Omega}$ ) should be slow enough, such that by the time $\tau_{\text {ent }}$ it takes to ramp above $q_{\mathrm{c}}$, the information is not lost. In practice, this can be achieved by switching $q$ very fast, at a time $\tau_{\mathrm{ent}} \ll \Gamma_{\mathrm{r}, \max }^{-1}$, to $q_{\mathrm{c}}$ and slower afterwards, when the actual measurement occurs.

In the ohmic spin-boson model, ${ }^{9,16}$ i.e., for $J_{\text {eff }}$ $=2 \pi \alpha \omega e^{-\omega / \omega_{c}}$, a scaling of $\Delta_{\text {eff }}$ to zero can be achieved through a dissipative phase transition at strong coupling to the bath $(\alpha>1){ }^{9,17,24,25}$ This transition is driven by the entanglement with a collective state involving the whole oscillator bath. Ramping $\alpha$ to large values increases $J_{\text {eff }}(\omega)$ at all frequencies, which leads to rapid relaxation before the scaling is established. Moreover, it is not known, how long it will take for the system to go through this phase transition. Here, according to the adiabatic theorem, this time is set through the inverse level spacing of the coupled system, which is infinite for the dense Ohmic spectrum. On the contrary, the model studied in the present paper provides strong scaling of $\Delta_{\text {eff }}$ with predictably slow relaxation and gives a clear prediction for the time scale of the entanglement set by the finite level spacing.

This model does not generally predict the efficiency of the detection. In order to do so, I chose a specific realization of the model, a superconducting quantum bit. ${ }^{5,6}$ In this case, the readout device is a Josephson junction, whose critical current $I_{0}$ is influenced by the state of the qubit, either a superconducting single-electron transistor ${ }^{5}$ or a dc superconducting quantum interference device (SQUID), ${ }^{6}$ see Fig. 2 . We study the junction on the superconducting branch at low bias current $I_{\mathrm{B}}$. We assume the tunable junction to be shunted only by a very large resistor $R$ and an external capacitance $C_{\mathrm{x}}$ and consequently underdamped. This system has been studied in

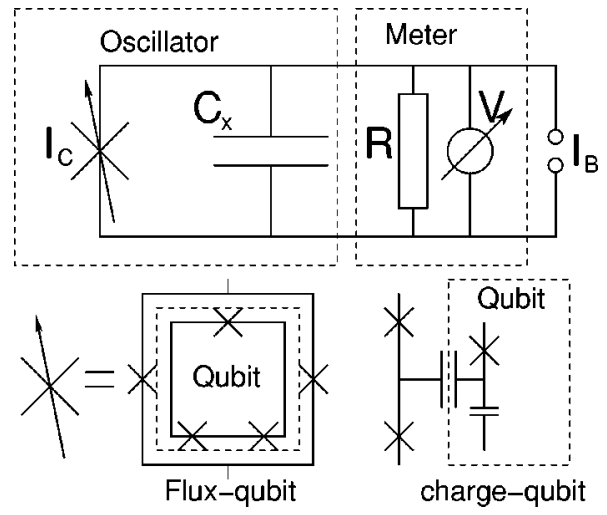

FIG. 2. Underdamped readout devices for superconducting flux (left) and charge (right) quantum bits.

the weak damping regime in Ref. 8 and resembles the one experimentally used in (Ref. 4). The oscillator in our model represents the plasma resonance ${ }^{26}$ of the tunable junction at $\Omega=\sqrt{2 e I_{0} / \hbar C_{\mathrm{x}}}\left(1-I_{\mathrm{B}}^{2} / I_{0}^{2}\right)^{1 / 4}$. We can identify $M$ $=C_{\mathrm{X}}(\hbar / 2 e)^{2}, \quad X=\phi \quad$ (the Josephson phase), and $q$ $=\left(\delta I_{c} / I_{c}\right)\left(I_{B} / \sqrt{I_{0}^{2}-I_{B}^{2}}\right)$, where $\delta I_{c} / I_{c}$ is the difference in critical current induced by the two fundamental states of the qubit. The damping is provided by the resistor and leads to $\alpha=h q^{2} / 2 e^{2} R=q^{2} 11.8 k \Omega / R$, and the scaling exponent reads $\eta=q^{2} \sqrt{C_{\mathrm{x}} I_{c} \hbar / 8 e^{3}}$.

When ramping $I_{\mathrm{B}}$, the junction switches to a finite voltage at $I_{\mathrm{sw}}<I_{0}$, which provides a measure for $I_{0}$. This switching is a stochastic process, so, if the measurement is repeated, one finds a histogram of switching currents $^{27,28}$ centered around $I_{\mathrm{sw}, 0}$, whose width $\delta I_{\mathrm{sw}}$ limits the resolution of this detector. In our case, the switching is predominantly due to thermal activation, where we can express $I_{\mathrm{sw}, 0} / I_{0}$ $=1-\left[\ln \left(\omega_{T} / \Gamma_{S}\right) / u_{0}\right]^{2 / 3}$ and $\delta I / I_{0}=\left[u_{0}^{2} \ln \left(\omega_{T} / \Gamma_{S}\right)\right]^{-1 / 3}$ through the dimensionless height of the barrier at zero bias $u_{0}$ $=(4 \sqrt{2} / 3)\left(\hbar I_{c} / 2 e k T\right)$, the activation frequency $\omega_{\mathrm{T}}$ $=2 \Omega / \pi$, and the ramp rate $\Gamma_{s}=d\left(q / q_{\max }\right) / d t$. The current can be switched within a time $\tau_{\mathrm{sw}}=\Omega^{-1}$, i.e., the ramp rate is limited by $\Gamma_{\mathrm{s}, \max }=\Omega$.

In a flux qubit, one can realize $I_{\mathrm{c}}=1 \mu \mathrm{A}$, shunt with $C_{\mathrm{x}}=100 \mathrm{pF}$ and $R=10 \mathrm{k} \Omega$, and $q_{\max }=0.05$ at a typical switching current level. We will assume $\Delta_{0}=1 \mathrm{GHz}$ and $\epsilon$ $=1 \mathrm{GHz}$ for the qubit. These parameters are accessible by doubling the size of the sample studied in (Ref. 4). This leads to $\Omega=2 \mathrm{GHz}, \quad \alpha=0.003$, and $\eta_{\max }=3.5$, i.e., $\Delta_{\text {eff }}$ $=0.03 \Delta_{0}$. Entanglement sets in at $q_{\mathrm{c}}=0.015$, where the relaxation time is $\tau_{\mathrm{r}, \min }=\Gamma_{\mathrm{r}, \max }^{-1}=5 \mu \mathrm{s}$. For $1 \%$ error, the first switch over this point has to be done at $700 \mathrm{~ns}$, which is way above $\Gamma_{\mathrm{s}, \max }=500 \mathrm{ps}$ and the adiabatic condition $q d q / d t$ $<(500 \mathrm{~ns})^{-1}$. Close to the measuring point $I_{\mathrm{sw}, 0}$, we find $\tau_{\mathrm{r}}=120 \mu \mathrm{s}$ and $\tau_{\phi}=100 \mathrm{~ns}$, which leaves a huge measurement window.

For definiteness, we set the temperature to $T=200 \mathrm{mK}$ and find, using $\Gamma_{s}=(15 \mu \mathrm{s})^{-1}$, that $I_{\mathrm{sw}, 0} / I_{0}=0.96$ and $\delta I / I_{0}=0.35 \%$, so, because $q=5 \%$, we have a signal-tonoise ratio of about 14. Hence, a single-shot von Neumann measurement appears to be feasible within a gradual improvement of technology. 
For the readout for a charge qubit ${ }^{3}$ by a superconducting single electron transistor (S-SET), one can achieve values of $q=0.5$ within a charging energy $E_{\mathrm{C}, \mathrm{SET}}=2 \mathrm{~K}$, corresponding to a capacitance scale of $C=1 \mathrm{fF}$. We take the critical current of the SET to be $I_{\mathrm{c}}=10 \mathrm{nA}$ and a shunt of $R=10 \mathrm{k} \Omega$ and $C_{\mathrm{x}}=1 \mathrm{pF}$ shunt capacitance. This leads to $\eta=3.5, \Omega$ $=2 \mathrm{GHz}$, and damping $\alpha=0.25$. Assuming $\Delta=1 \mathrm{GHz}$, and $\epsilon=1 \mathrm{GHz}$, we find $\tau_{\mathrm{R}, \min }=60 \mathrm{~ns}$, so for $1 \%$ error we have to switch to $q_{c}$ in about $10 \mathrm{~ns}$, which is close to the limit of $\Gamma_{\mathrm{s}, \max }=500 \mathrm{ps}$. however, may pose some challenge for the limiting time scales which are not due to the on-chip circuitry. For the readout step, we find $\tau_{\mathrm{R}}=25 \mu \mathrm{s}$ and $\tau_{\phi}$ $=15 \mathrm{~ns}$. Applying the histogram theory as above at $T$ $=200 \mathrm{mK}$ and $\Gamma_{\mathrm{S}}=(3 \mu \mathrm{s})^{-1}$, we end up with $I_{\mathrm{sw}, 0} / I_{0}$ $=40 \%$ and $\delta I_{\mathrm{sw}} / I_{\mathrm{c}}=8 \%$, which can resolve our large signal of $q=30 \%$ at signal-to-noise ratio of 4 . It has been shown ${ }^{29,30}$ that experimentally SET's can reach signal-tonoise figures comparable to the quantum limit, hence even though the resolution is slightly less favorable than above, von Neumann measurements appear to be possible. For qubits $^{31}$ operating in the regime of $E_{J} / E_{c} \simeq 1$, more favorable parameters should be accessible.

The readout of the detector by switching is only one, and not necessarily the optimum alternative. Measurements could also be performed by detecting the kinetic inductance using the same parameters.

A similar circuit, a normal conducting SET with out the shunt capacitor has been thoroughly studied in Refs. 5,7. In that case, the measurement is started by rapidly switching the gate and monitoring the current. It has been shown that in this way weak as well as strong measurements can be performed. As a consequence of the direct coupling of a dense spectrum of normal electrons to the qubit, the entanglement and the decoherence are not as strongly separated as in our case. Typical ${ }^{5}$ mixing times during the whole measurement are of the order of $1 \mu \mathrm{s}$, i.e., one has to be able to monitor the current through the SET on the scale of $100 \mathrm{~ns}$. In our case, one has to make the first entanglement switch on a similar scale, but has to be monitor the voltage only afterwards, when mixing times are on the scale of $10 \mu \mathrm{s}$. These numbers clearly indicate an advantage of the entanglement with the intermediate oscillator.

I have proposed a strategy for performing detectordominated von Neumann measurements on qubits, using entanglement with coherent states of an harmonic oscillator. This system has been quantitatively analyzed using the spinboson model and it has been shown that it has very favorable coherence and relaxation properties. A connection between the familiar scaling of the tunnel matrix element and the degree of entanglement with the environment has been established. Realistic superconducting circuitry, which could perform such measurements within present-day technology, has been proposed.

I thank J. von Delft, M. Governale, M. Grifoni, A.C.J. ter Haar, P. Hadley, P. Hakonen, C.J.P.M. Harmans, S. Kleff, L. Levitov, S. Lloyd, A. Lupascu, J.E. Mooij, T.P. Orlando, A. Shnirman, and C.H. van der Wal for clarifying discussions, as well as acknowledge support by the EU through TMR Supnan and Quiprocone and through ARO under Contract No. P-43385-PH-QC.
${ }^{1}$ See, e.g., M.A. Nielsen and I.L. Chuang, Quantum Computation and Quantum Information (Cambridge University Press, Cambridge, 2000).

${ }^{2}$ J. von Neumann, Mathematical Foundations of Quantum Mechanics (Princeton University Press, Princeton, NJ, 1955).

${ }^{3}$ Y. Nakamura, Yu.A. Pashkin, and J.S. Tsai, Nature (London) 398, 786 (1999).

${ }^{4}$ C.H. van der Wal et al., Science 290, 773 (2000); I. Chiorescu et al., ibid. 299, 1869 (2003).

${ }^{5}$ Yu. Makhlin, G. Schön, and A. Shnirman, Nature (London) 386, 305 (1999); Rev. Mod. Phys. 73, 357 (2001).

${ }^{6}$ J.E. Mooij et al., Science 285, 1036 (1999).

${ }^{7}$ A. Shnirman and G. Schön, Phys. Rev. B 57, 15400 (1998).

${ }^{8}$ C.H. van der Wal et al., Eur. Phys. J. B 31, 111 (2003).

${ }^{9}$ A.J. Leggett et al., Rev. Mod. Phys. 59, 1 (1987).

${ }^{10}$ M. Grifoni, E. Paladino, and U. Weiss, Eur. Phys. J. B 10, 719 (1999)

${ }^{11}$ A.N. Korotkov and D.V. Averin, Phys. Rev. B 64, 165310 (2001).

${ }^{12}$ D.V. Averin, Exploring the Quantum/Classical Frontier, edited by J.R. Friedman and S. Han (Nova, Commack, NY, 2002).

${ }^{13}$ W.H. Zurek, Phys. Rev. D 24, 1516 (1981); Prog. Theor. Phys. 89, 281 (1993).

${ }^{14}$ P.W. Shor, Phys. Rev. A 52, R2493 (1995).

${ }^{15}$ A.J. Leggett and A. Garg, Phys. Rev. Lett. 54, 857 (1985).
${ }^{16}$ See, e.g., U. Weiss, Quantum Dissipative Systems, 2nd ed. (World Scientific, Singapore, 1999).

${ }^{17}$ G.T. Zimanyi and K. Vladar, Phys. Rev. A 34, 3496 (1986).

${ }^{18}$ T.P. Orlando et al., Phys. Rev. B 60, 15398 (1999).

${ }^{19}$ A. Garg, J.N. Onuchic, and V. Ambegaokar, J. Chem. Phys. 83, 4491 (1985).

${ }^{20}$ This nonseparable state is not fully entangled, the degree of entanglement is $1-e^{-\eta}$.

${ }^{21}$ A. Peres, Quantum Theory: Concepts and Methods (Kluwer, Dordrecht, 1993).

${ }^{22}$ S. Chakravarty, Phys. Rev. Lett. 49, 681 (1982).

${ }^{23}$ F.K. Wilhelm, S. Kleff, and J. von Delft (unpublished).

${ }^{24}$ A. Schmid, Phys. Rev. Lett. 51, 1506 (1983); see also G. Schön and A.D. Zaikin, Phys. Rep. 198, 237 (1990).

${ }^{25}$ J.S. Penttilä et al., Phys. Rev. Lett. 82, 1004 (1999).

${ }^{26}$ For an introduction to Josephson-junction physics, see, e.g., M. Tinkham, Introduction to Superconductivity, 2nd ed. (McGrawHill, New York, 1996).

${ }^{27}$ R.F. Voss and R.A. Webb, Phys. Rev. Lett. 47, 265 (1981).

${ }^{28}$ A summary of this field is found in J.M. Martinis, M.H. Devoret, and J. Clarke, Phys. Rev. B 35, 4682 (1987).

${ }^{29}$ M.H. Devoret and R.J. Schoellkopf, Nature (London) 406, 1039 (2000).

${ }^{30}$ D. Vion et al., Phys. Rev. Lett. 77, 3435 (1996).

${ }^{31}$ D. Vion et al., Science 296, 886 (2002). 